\title{
GENERALIZED AND CLASSICAL SOLUTIONS OF THE NONLINEAR STATIONARY NAVIER-STOKES EQUATIONS
}

BY

\author{
VICTOR L. SHAPIRO $\left({ }^{1}\right)$
}

ABSTRACT. New regularity results in domains of Euclidean 3-space are established for the generalized solutions of the nonlinear stationary Navier-Stokes equations in terms of Dini criteria on the external force.

1. Introduction. Let $\Omega$ be a domain (bounded or unbounded) in Euclidean 3-space $E_{3}$, and let $\mathbf{f}=\left(f_{1}, f_{2}, f_{3}\right)$ be a fixed continuous vector defined in $\Omega$. The pair $\mathbf{v}, p$ will be said to be a classical solution of the nonlinear stationary Navier-Stokes equations if $\mathrm{v}$ is in $C^{2}(\Omega), p$ is in $C^{1}(\Omega)$, and

$$
\nu \Delta \mathbf{v}-(\mathbf{v} \cdot \nabla) \mathbf{v}-\nabla p=-\mathbf{f}, \quad \operatorname{div} \mathbf{v}=0
$$

in $\Omega$ where $\nu$ is a positive constant.

Following the notation in [4], we shall designate by $j(\Omega)$ the set of vectors which are in $C^{\infty}(\Omega)$, solenoidal in $\Omega$, and which vanish outside of a compact subset of $\Omega$ : In $j(\Omega)$ we introduce the inner product

$$
[\mathrm{w}, \mathrm{v}]=\int_{\Omega} \sum_{k=1}^{3} \mathrm{w}_{x_{k}} \cdot \mathrm{v}_{x_{k}} d x
$$

where $\mathbf{w} \cdot \mathbf{v}$ designates the usual dot product

$$
\mathbf{w} \cdot \mathbf{v}=\sum_{j=1}^{3} w_{j} v_{j}
$$

By $H(\Omega)$ we shall designate the Hilbert space which is the closure of $j(\Omega)$ under the norm induced by (1.2).

We shall say $\mathbf{v}$ is a generalized solution of the nonlinear stationary NavierStokes equations if $\mathbf{v}$ is in $H(\Omega)$, if $\mathbf{f}$ is locally in $L^{1}(\Omega)$, and if

$$
\sum_{k=1}^{3} \int_{\Omega}\left(\nu \mathbf{v}_{x_{k}} \cdot \phi_{x_{k}}-v_{k} \mathbf{v} \cdot \phi_{x_{k}}\right) d x=\int_{\Omega} \mathbf{f} \cdot \phi d x \text { for all } \phi \text { in } \dot{J}(\Omega) \text {. }
$$

Received by the editors January 11, 1974.

AMS (MOS) subject classifications (1970). Primary 76D05, 35Q10; Secondary 35D10.

Key words and phrases. Stationary Navier-Stokes equations, generalized solutions, classical solutions, nonlinear partial differential equations, Dini condition.

( $\left.{ }^{1}\right)$ Research sponsored by the Air Force Office of Scientific Research, Office of Aerospace Research, USAF, under Grant No. AFOSR 73-2456. The United States Government is authorized to reproduce and distribute reprints for governmental purposes notwithstanding any copyright notation hereon. 
Next, we let $\Omega_{1}$ designate a subdomain of $\Omega$ whose closure is compact in $\Omega$, and we denote the diameter of $\Omega_{1}$ by $\delta\left(\Omega_{1}\right)$. Also, we suppose $f$ is in $C^{0}\left(\bar{\Omega}_{1}\right)$. Then for $0<t \leqslant \delta\left(\Omega_{1}\right)$, we set

$$
\omega\left(t, \mathbf{f}, \Omega_{1}\right)=\sup _{|x-y| \leqslant t ; x, y \operatorname{in} \Omega_{1}}|\mathbf{f}(x)-\mathbf{f}(y)| .
$$

We shall say $\mathbf{f}$ satisfies a Dini condition locally in $\Omega$ if $\mathbf{f}$ is in $C^{0}(\Omega)$ and if for every $\Omega_{1} \subset \Omega$, with $\Omega_{1}$ as above, the following holds:

$$
\int_{0}^{\delta\left(\Omega_{1}\right)} \omega\left(t, \mathrm{f}, \Omega_{1}\right) t^{-1} d t \text { is finite. }
$$

Next, we let $\Omega_{2}$ be a subdomain of $\Omega_{1}$ whose closure is compact in $\Omega_{1}$ and assume that (1.5) holds. We shall say $g$ is in $C^{\omega^{*}\left(t, f, \Omega_{1}\right)}\left(\Omega_{2}\right)$ if there is a constant $A$ such that for $x, y$ in $\Omega_{2}$,

$$
|g(x)-g(y)| \leqslant A \omega^{*}\left(|x-y|, \mathbf{f}, \Omega_{1}\right)
$$

where for $0<t \leqslant \delta\left(\Omega_{1}\right)$,

$$
\omega^{*}\left(t, \mathbf{f}, \Omega_{1}\right)=\int_{0}^{t} \omega\left(s, \mathbf{f}, \Omega_{1}\right) s^{-1} d s+t \int_{t}^{\delta\left(\Omega_{1}\right)} \omega\left(s, \mathbf{f}, \Omega_{1}\right) s^{-2} d s .
$$

For future reference we note that an integration by parts also gives the representation

$$
\omega^{*}\left(t, \mathbf{f}, \Omega_{1}\right)=\int_{0}^{t} \int_{s}^{\delta\left(\Omega_{1}\right)} \omega(r) r^{-2} d r d s
$$

We observe at this juncture from (1.7) that (1.5) implies that $\omega^{*}\left(t, f, \Omega_{1}\right) \rightarrow$ 0 as $t \rightarrow 0$. Also we note that if $\mathrm{f}$ is in $C^{\theta}\left(\Omega_{1}\right), 0<\theta<1$, and $g$ is in $C^{\omega^{*}\left(t, \mathrm{f}, \Omega_{1}\right)}\left(\Omega_{2}\right)$, then $g$ is in $C^{\theta}\left(\Omega_{2}\right)$. Furthermore, we observe that if there is constant $A^{\prime}$ such that for $x, y$ in $\Omega$,

$$
|\mathrm{f}(x)-\mathrm{f}(y)| \leqslant A^{\prime}|\log | x-y||^{-(1+\theta)}, \quad 0<\theta<1,
$$

then $g$ in $C^{\omega^{*}\left(t, \mathrm{f}, \Omega_{1}\right)}\left(\Omega_{2}\right)$ implies that there is a constant $A$ such that

$$
|g(x)-g(y)| \leqslant A|\log | x-y||^{-\theta} \text { for } x, y \text { in } \Omega_{2} \text {. }
$$

$g$ in $C^{1+\omega^{*}\left(t, \mathrm{f}, \Omega_{1}\right)}\left(\Omega_{2}\right)$ will mean that $g$ is in $C^{1}\left(\Omega_{2}\right)$ and $g_{x_{j}}$ is in $C^{\omega^{*}\left(t, \mathrm{f}, \Omega_{1}\right)}$, $j=1,2,3 . g$ in $C^{2+\omega^{*}\left(t, f, \Omega_{1}\right)}\left(\Omega_{2}\right)$ will be defined in a similar manner using the second derivatives of $g$.

In this paper, we intend to establish the following results:

THEOREM 1. Let $\Omega$ be a domain contained in $E_{3}$. Suppose that $\mathbf{f}$ satisfies a Dini condition locally in $\Omega$. Suppose furthermore that $\mathbf{v}$ is in $H(\Omega)$ and is also a generalized solution of the nonlinear stationary Navier-Stokes equations, i.e., $\mathbf{v}$ satisfies (1.3). Then the following hold:

(i) there is $a \mathbf{v}^{\prime}$ in $C^{2}(\Omega)$ such that $\mathbf{v}^{\prime}=\mathbf{v}$ almost everywhere in $\Omega$;

(ii) there is a $p$ in $C^{1}(\Omega)$ such that the pair $\mathrm{v}^{\prime}, p$ is a classical solution of the nonlinear stationary Navier-Stokes equations, i.e., v', $p$ satisfies (1.1); 
(iii) if $\Omega_{1}$ and $\Omega_{2}$ are bounded subdomains of $\Omega$ with $\bar{\Omega}_{2} \subset \Omega_{1} \subset \bar{\Omega}_{1} \subset \Omega$ and if $\mathbf{f}$ is not identically constant in $\Omega_{1}$, then $\mathbf{v}^{\prime}$ and $p$ are in $C^{i+\omega^{*}\left(t, \mathbf{f}, \Omega_{1}\right)}\left(\Omega_{2}\right)$ where $i=2$ and 1 , respectively.

THEOREM 2. Let $\Omega$ be a domain contained in $E_{3}$, and let $\Omega^{\prime}$ be a subdomain contained in $\Omega$. Suppose that $\mathbf{f}=\left(f_{1}, f_{2}, f_{3}\right)$ is locally in $L^{2}(\Omega)$ and is in $C^{n}\left(\Omega^{\prime}\right), n$ a nonnegative integer. Suppose also that for $k=1,2,3$, every partial derivative of order $n$ of $f_{k}$ satisfies a Dini condition locally in $\Omega^{\prime}$. Suppose furthermore that $\mathbf{v}$ is in $H(\Omega)$ and is also a generalized solution of the nonlinear stationary Navier-Stokes equations. Then the following hold:

(i) there is $a \mathbf{v}^{\prime}$ in $C^{n+2}\left(\Omega^{\prime}\right)$ such that $\mathbf{v}^{\prime}=\mathbf{v}$ almost everywhere in $\Omega^{\prime}$;

(ii) there is a $p$ in $C^{n+1}\left(\Omega^{\prime}\right)$ such that the pair $\mathbf{v}^{\prime}, p$ is a classical solution of the nonlinear stationary Navier-Stokes equations in $\Omega^{\prime}$.

In (iii) of Theorem 1 above, if $\mathbf{f}$ is identically constant in $\Omega_{1}$, then it follows from Theorem 2 that $\mathbf{v}^{\prime}$ and $p$ are in $C^{\infty}\left(\Omega_{1}\right)$. Theorem 1 above is to be viewed as giving an extension of the second part of [4, Theorem 6, p. 131], and Theorem 2 above as giving an extension of the second part of [2, Theorem 4.2, p. 79].

In Euclidean 2-space, the analogues of Theorems 1 and 2 above are also valid.

2. Stokes flow and multiple Fourier series. To establish the above theorem, we shall need some lemmas connecting multiple Fourier series with the equations giving rise to a Stokes flow, namely

$$
v \Delta \mathbf{v}-\nabla p=\mathbf{f}, \quad \operatorname{div} \mathbf{v}=0 .
$$

We shall use the following notation: $T_{3}=\left\{x:-\pi \leqslant x_{j}<\pi, j=1,2,3\right\} ; m$ will designate an integral lattice point; for a function $g$ in $L^{1}\left(T_{3}\right)$, we shall set

$$
g^{\wedge}(m)=(2 \pi)^{-3} \int_{T_{3}} g(x) e^{-i(m, x)} d x .
$$

Also, $(x, y)=x_{1} y_{1}+x_{2} y_{2}+x_{3} y_{3}$ will designate the usual inner product; $(x, x)^{1 / 2}$ will be designated by $|x| ; B(x, r)$ will designate the open 3-ball with center $x$ and radius $r$, and $S(x, r)$ will designate its boundary. In particular, $S(0,1)$ will represent the unit 2-sphere $\{x:|x|=1\}$.

Next, we discuss the theory of periodic singular integrals. $K$ will be called a Calderón-Zygmund kernel if

$$
K(x)=Z(x /|x|)|x|^{-3} \text { for } 0<|x|<\infty,
$$

where

$$
Z \text { is a function in } C^{\gamma}[S(0,1)], \quad 0<\gamma \leqslant 1,
$$

and 


$$
\int_{S(0,1)} Z(\xi) d S(\xi)=0
$$

For $x$ in $E_{3}-\bigcup_{m \neq 0}\{2 \pi m\}$, we define

$$
K^{* *}(x)=\lim _{R \rightarrow \infty} \sum_{1 \leqslant|m| \leqslant R}[K(x+2 \pi m)-K(2 \pi m)],
$$

and observe from [1, pp. 251-252] that the series on the right-hand side of (2.6) converges uniformly on compact subsets of $E_{3}-U_{m \neq 0}\{2 \pi m\}$. In particular,

$$
K^{* *} \text { is a bounded continuous function on } T_{3} \text {. }
$$

Next, we observe from (2.3), (2.4), and (2.5) that

$$
\lim _{\epsilon \rightarrow 0} \int_{T_{3}-B(0, \epsilon)} K(x) d x \text { exists and is finite. }
$$

Using (2.7) and (2.8), we define the constant $c_{K}$ by

$$
c_{K}=(2 \pi)^{-3} \lim _{\epsilon \rightarrow 0} \int_{T_{3}-B(0, \epsilon)}\left[K(x)+K^{* *}(x)\right] d x,
$$

and set

$$
K^{*}(x)=K(x)+K^{* *}(x)-c_{K} \text { for } x \text { in } E_{3}-\bigcup_{m}\{2 \pi m\}
$$

We note that

$$
K^{*} \text { is a periodic function of period } 2 \pi \text { in }
$$

$$
\text { each variable in } E_{3}-\bigcup_{m}\{2 \pi m\} \text {. }
$$

Also, we note that

$$
\lim _{\epsilon \rightarrow 0} \int_{T_{3}-B(0, \epsilon)} K^{*}(x) d x=0 .
$$

For $g$ a continuous periodic function in $E_{3}$ (henceforth, periodic will mean periodic of period $2 \pi$ in each variable), we shall set

$$
\omega\left(t, g, T_{3}\right)=\sup _{|x-y| \leqslant t, x, y \text { in } E_{3}}|g(x)-g(y)|,
$$

and shall say (as before) that $g$ satisfies a Dini condition on $T_{3}$ if

$$
\int_{0}^{1} \omega\left(t, g, T_{3}\right) t^{-1} d t \quad \text { is finite. }
$$

Next, for $0<\gamma \leqslant 1$ and $0<t \leqslant 1$, we shall set

$$
\omega^{* *}(t, g, \gamma)=\int_{0}^{t} \omega\left(t, g, T_{3}\right) s^{-1} d s+t^{\gamma} \int_{t}^{1} \omega\left(s, g, T_{3}\right) s^{-(1+\gamma)} d s .
$$

We shall say $h$ is in $C^{\omega^{* *}(t, g, \gamma)}\left(T_{3}\right)$ if $h$ is a continuous periodic function in $E_{3}$ and if, furthermore, there is a constant $A$ such that for

$$
\begin{aligned}
& x, y \text { in } E_{3} \text { and }|x-y| \leqslant 1, \\
& \cdot|h(x)-h(y)| \leqslant A \omega^{* *}(|x-y|, g, \gamma) .
\end{aligned}
$$


With $K^{*}(x)$ defined by (2.10) and $g$ a periodic function in $E_{3}$ in $L^{1}\left(T_{3}\right)$, we shall designate the following limit, provided it exists, by

$$
\tilde{g}_{K^{*}}(x)=\lim _{\epsilon \rightarrow 0}(2 \pi)^{-3} \int_{T_{3}-B(0, \epsilon)} g(x-y) K^{*}(y) d y .
$$

The first lemma we intend to establish is the following:

LEMMA 1. Suppose that $g$ is a continuous periodic function satisfying a Dini condition on $T_{3}$. Then $\widetilde{g}_{K^{*}}(x)$ exists for every $x$ and is in $C^{\omega^{* *}(t, g, \gamma)}\left(T_{3}\right)$.

For the 1-dimensional analogue of the above result, we refer the reader to [6, p. 121].

Since $g$ meets a Dini condition on $T_{3}$ and since $Z(\xi)$ is uniformly bounded for $|\xi|=1$, it follows immediately from (2.3) that for every $x$,

$$
\int_{B(0,1)}|g(x-y)-g(x)||K(y)| d y<+\infty
$$

But then as a consequence of this fact, (2.10), (2.12), and (2.17), we have that $\tilde{g}_{K^{*}}$ exists and is finite for every $x$ and equals

$$
\tilde{g}_{K^{*}}(x)=(2 \pi)^{-3} \int_{T_{3}}[g(x-y)-g(x)] K^{*}(y) d y .
$$

Therefore for $|z|<10^{-1}$, we also have from the periodicity of $g$, (2.11), and (2.18) that

$$
\tilde{g}_{K^{*}}(x+z)=(2 \pi)^{-3} \int_{T_{3}}[g(x-y)-g(x+z)] K^{*}(y+z) d y
$$

$$
\text { for } x \text { in } T_{3} \text {. }
$$

Next, we set

$$
I(s)=\int_{B(0, s)} \omega\left(|x|, g, T^{3}\right)\left|K^{*}(x)\right| d x,
$$

and observe from (2.19) and (2.20) that for $|z|<10^{-1}$ and $x$ in $T_{3}$,

$$
\begin{aligned}
(2 \pi)^{3}\left|\tilde{g}_{K^{*}}(x+z)-\tilde{g}_{K^{*}}(x)\right| & \\
\leqslant & \left|\int_{T_{3}-B(0,3|z|)}[g(x-y)-g(x)]\left[K^{*}(y+z)-K^{*}(y)\right] d y\right| \\
& +|g(x+z)-g(x)|\left|\int_{T_{3}-B(0,3|z|)} K^{*}(y+z) d y\right|+2 I(4|z|) .
\end{aligned}
$$

Now it is clear from the observation made after (2.6) that there is a constant $c_{K, 1}$ such that

$$
\left|K^{* *}(x+z)\right| \leqslant c_{K, 1} \text { for } x \text { in } T_{3} \text { and }|z|<10^{-1} .
$$

Letting $T_{3}+z=\left\{y: y=x+z, x\right.$ in $\left.T_{3}\right\}$, we see from (2.3) and (2.4) that there is a constant $c_{K, 2}$ such that 


$$
\begin{aligned}
\left|\left\{\int_{\left(T_{3}+z\right)-B(z, 3|z|)}-\int_{T_{3}-B(z, 3|z|)}\right\} K(y) d y\right| & \leqslant c_{K, 2}|z| \\
& \text { for }|z|<10^{-1} .
\end{aligned}
$$

Also, we note that there is a constant $c_{K, 3}$ such that

$$
\int_{2|z| \leqslant|y| \leqslant 4|z|}|K(y)| d y \leqslant c_{K, 3} \text { for }|z|<10^{-1} .
$$

Consequently,

$$
\begin{aligned}
&\left|\left\{\int_{T_{3}-B(z, 3|z|)}-\int_{T_{3}-B(0,3|z|)}\right\} K(y) d y\right| \leqslant c_{K, 3} \\
& \text { for }|z|<10^{-1} .
\end{aligned}
$$

Observing that $\lim _{\epsilon \rightarrow 0} \int_{T_{3}-B(0, \epsilon)} K(y) d y$ exists and is finite, we obtain from (2.10), (2.23), (2.24), and (2.25) that there is a constant $c_{K, 4}$ such that

$$
\left|\int_{T_{3}-B(0,3|z|)} K^{*}(y+z) d y\right| \leqslant c_{K, 4} \text { for }|z|<10^{-1} .
$$

Next, from (2.3) and (2.4), we observe (as in [1, p. 263, 1. 17]) that there is a $c_{K, 5}$ such that

$$
|K(y+z)-K(y)| \leqslant c_{K, 5}|z|^{\gamma}|y|^{-(3+\gamma)}
$$

$$
\text { for } 3|z| \leqslant|y|<\infty \text { and }|z|<10^{-1} \text {. }
$$

Also, from (2.6) and (2.27), we observe that there is a constant $c_{K, 6}$ such that

$$
\left|K^{* *}(y+z)-K^{* *}(y)\right| \leqslant c_{K, 6}|z|^{\gamma}
$$

$$
\text { for } y \text { in } T_{3}-B(0,3|z|) \text { and }|z|<10^{-1} \text {. }
$$

As a consequence of (2.27), (2.28), and (2.10), we obtain that the first term on the right-hand side of the inequality in (2.22) is majorized by

$$
\begin{aligned}
c_{K, 5}|z|^{\gamma} & \int_{T_{3}-B(0,3|z|)} \lg (x+y)-\left.g(x)|| y\right|^{-(3+\gamma)} d y \\
& \quad+c_{K, 6}|z|^{\gamma} \int_{T_{3}-B(0,3|z|)}|g(x+y)-g(x)| d y .
\end{aligned}
$$

From (2.22), (2.26), and this last fact, we conclude that there is a constant $c_{K, 7}$ such that

$$
\begin{array}{ll}
\sup _{x \sin T_{3}} & \widetilde{g}_{K^{*}}(x+z)-\tilde{g}_{K^{*}}(x) \mid \\
\quad \leqslant c_{K, 7}\left[\omega\left(|z|, g, T_{3}\right)+I(4|z|)+|z|^{\gamma} \int_{T_{3}-B(0,3|z|)} \omega\left(|y|, g, T_{3}\right)|y|^{-(3+\gamma)} d y\right] \\
(2.29) & \text { for }|z|<10^{-1} .
\end{array}
$$


From (2.3), (2.4), (2.7), and (2.10), we see that there is a constant $c_{K, 8}$ such that

$$
\left|K^{*}(x)\right| \leqslant c_{K, 8}|x|^{-3} \text { for } 0<|x| \leqslant 1 .
$$

Consequently, we infer from (2.29) and (2.30) that there is a constant $c_{K, 9}$ such that

$$
\begin{aligned}
\sup _{x \sin T_{3}} \tilde{g}_{K^{*}}(x+z)-\tilde{g}_{K^{*}}(x) \mid & \\
\leqslant c_{K, 9}\left[\int_{0}^{|z|} \omega\left(t, g, T_{3}\right) t^{-1} d t+|z|^{\gamma} \int_{|z|}^{1} \omega\left(t, g, T_{3}\right) t^{-(1+\gamma)} d t\right] & \quad \text { for }|z|<10^{-1} .
\end{aligned}
$$

From (2.31) and the hypothesis of the lemma, we conclude immediately that $\tilde{g}_{K^{*}}$ is a continuous periodic function. Next, we observe that the expression in brackets on the right-hand side of the inequality in (2.31) is an increasing function of $|z|$ for $0<|z|<1$. Consequently, we infer from (2.15) and (2.31) that

$$
\begin{aligned}
& \left|\tilde{g}_{K^{*}}(x)-\tilde{g}_{K^{*}}(y)\right| \leqslant 10 c_{K, 9} \omega^{* *}(|x-y|, g, \gamma) \\
& \qquad \text { for } x, y \text { in } E_{3} \text { and }|x-y| \leqslant 1 .
\end{aligned}
$$

Therefore, $\widetilde{g}_{K^{*}}$ is in $C^{\omega^{* *}(t, g, \gamma)}\left(T_{3}\right)$, and the proof of the lemma is complete.

The next lemma we establish is the following:

LEMMA 2. Suppose that $g$ is a continuous periodic function satisfying $a$ Dini condition on $T_{3}$. Then for $j, k, \alpha, \beta=1,2,3$, there are functions $g_{j k}$ and $g_{j k \alpha \beta}$ in $C^{\omega^{* *}(t, g, 1)}\left(T_{3}\right)$ with $g_{j k}(0)=g_{j k \alpha \beta}(0)=0$ such that for $m \neq 0$,

$$
\hat{g_{j k}}(m)=m_{j} m_{k}|m|^{-2} \hat{g^{\wedge}}(m)
$$

and

$$
\hat{g}_{j k \alpha \beta}(m)=m_{j} m_{k} m_{\alpha} m_{\beta}|m|^{-4} \hat{g^{\gamma}(m)} \text {. }
$$

We shall establish the above lemma for $g_{j k \alpha \beta}$, i.e., we shall show (2.33) holds. A similar proof will prevail for $g_{j k}$ to show that (2.32) holds.

In order to accomplish the assertion concerning $g_{j k \alpha \beta}$, we recall the definition of a spherical harmonic polynomial of order $n, n$ a nonnegative integer. In particular, $Y_{n}(x)$ is called a spherical harmonic polynomial of order $n$ if $Y_{n}$ is a homogeneous polynomial of degree $n$ with the added fact that $\Delta Y_{n}(x)=0$ for all $x$. ( $Y_{n} \equiv 0$ will also be called a spherical harmonic of order $n$.)

Next fix $j, k, \alpha, \beta$. Then it follows from [3, p. 147] that there are spherical harmonic polynomials $Y_{4}$ and $Y_{2}$ and a constant $c$ such that for every $x$,

Consequently,

$$
x_{j} x_{k} x_{\alpha} x_{\beta}=Y_{4}(x)+|x|^{2} Y_{2}(x)+|x|^{4} c .
$$

$$
m_{j} m_{k} m_{\alpha} m_{\beta}=Y_{4}(m)+|m|^{2} Y_{2}(m)+|m|^{4} c
$$


Comparing the right-hand side of (2.33) with (2.34), we see that (2.33) will be established once we show the following:

There are functions $h$ and $h^{\prime}$ in $C^{\omega^{* *}(t, g, 1)}\left(T_{3}\right)$

(2.35) with $\hat{h}(0)=h^{\prime \wedge}(0)=0$ such that for $m \neq 0$,

$h^{\wedge}(m)=Y_{4}(m)|m|^{-4} g^{\wedge}(m)$ and $h^{\prime \wedge}(m)=Y_{2}(m)|m|^{-2} g^{\wedge}(m)$.

We shall establish (2.35) for $h$. A similar proof will work for $h^{\prime}$. In order to do this, we set

$$
K(x)=Y_{4}(x)|x|^{-7} \text { for } x \neq 0 .
$$

Since $\int_{S(0,1)} Y_{4}(\xi) d S(\xi)=0$, we see from (2.3), (2.4), and (2.5) that

$K$ is a Calderón-Zygmund kernel where $Z(x /|x|)=Y_{4}(x)|x|^{-4}$ and $Z$ is in $C^{1}[S(0,1)]$.

Consequently from Lemma 1 and (2.37) we have, with $\tilde{g}_{K^{*}}$ defined by (2.17), that

$$
\widetilde{g}_{K^{*}} \text { is in } C^{\omega^{* *}(t, g, 1)}\left(T_{3}\right) .
$$

From [1, p. 259], we next observe that

$$
\tilde{g}_{K^{*}}^{\wedge}(m)=K^{* \wedge}(m) g^{\wedge}(m),
$$

where

$$
K^{* \wedge}(m)=(2 \pi)^{-3} \lim _{\epsilon \rightarrow 0} \int_{T_{3}-B(0, \epsilon)} e^{-i(m, x)} K^{*}(x) d x .
$$

From (2.12) we obtain that

$$
K^{* \wedge}(0)=0,
$$

and from [1, pp. 257-261] that there is a nonzero constant $c^{\prime}$ such that

$$
K^{* \wedge}(m)=c^{\prime} Y_{4}(m)|m|^{-4} \text { for } m \neq 0 \text {. }
$$

We set $h(x)=\tilde{g}_{K^{*}}(x) / c^{\prime}$ and observe from (2.38) that $h$ is in $C^{\omega^{* *}(t, g, 1)}\left(T_{3}\right)$ and from (2.39), (2.40), and (2.41) that $\hat{h}^{\wedge}(0)=0$ and $\hat{h}^{\wedge}(m)=Y_{4}(m)|m|^{-4} g^{\wedge}(m)$ for $m \neq 0$. Consequently (2.35) is established for $h(x)$, and the proof of the lemma is complete.

Next, for the sake of completeness, we establish the following remark.

REMARK 1. Let $g$ and $g_{j}$ be continuous periodic functions, $j=1,2,3$. Suppose that $\hat{g_{j}} \hat{(m)}=i m_{j} \hat{g^{\prime}}(m)$ for every lattice point $m$. Then $g$ is in $C^{1}\left(E_{3}\right)$ and $\partial g / \partial x_{j}=g_{j}$.

We prove the above remark by showing

$$
\partial g(x) / \partial x_{1}=g_{1}(x) \text { for all } x .
$$

Since a similar proof will show that the analogue of $(2.42)$ holds for $j=2,3$, the establishing of (2.42) is equivalent to establishing the remark.

To do this, set, for $t>0$, 


$$
g(x, t)=\sum_{m} \hat{g}^{\hat{(}(m)} e^{i(m, x)-|m| t},
$$

and define $g_{1}(x, t)$ in an analogous manner using $g_{1}(m)$ instead of $\hat{g^{\prime}}(m)$. Consequently,

$$
\partial g(x, t) / \partial x_{1}=\sum_{m} i m_{1} \hat{g}^{\wedge}(m) e^{i(m, x)-|m| t},
$$

and we conclude from the hypothesis of the remark that

$$
\partial g(x, t) / \partial x_{1}=g_{1}(x, t) \text { for } t>0 \text { and every } x .
$$

As a consequence of (2.44), we have that for $s>0$,

$$
g\left(x_{1}+s, x_{2}, x_{3}, t\right)-g\left(x_{1}, x_{2}, x_{3}, t\right)=\int_{0}^{s} g_{1}\left(x_{1}+r, x_{2}, x_{3}, t\right) d r
$$

From [5, p. 56], we see that, respectively, $g(x, t)$ and $g_{1}(x, t)$ tend uniformly to $g(x)$ and $g_{1}(x)$ as $t \rightarrow 0$. We conclude from this fact and (2.45) that

$$
g\left(x_{1}+s, x_{2}, x_{3}\right)-g\left(x_{1}, x_{2}, x_{3}\right)=\int_{0}^{s} g\left(x_{1}+r, x_{2}, x_{3}\right) d r .
$$

But this fact implies that (2.42) holds and the proof of Remark 1 is complete. In a similar manner, we observe that the following remark can be established.

REMARK 2. Let $g$ and $g_{j k}$ be continuous periodic functions, $j, k=1,2,3$. Suppose that $\hat{g}_{\hat{j} k}(m)=-m_{j} m_{k} \hat{g}(m)$ for every lattice point $m$. Then $g$ is in $C^{2}\left(E_{3}\right)$ and $\partial^{2} g / \partial x_{j} \partial x_{k}=g_{j k}$.

The proof of Remark 2 proceeds analogously to that of Remark 1 except that second differences are used. We leave the details of the proof of the reader.

Next, we introduce the functions

In particular,

$$
\begin{aligned}
& H, u_{j}^{k} \text {, and } q_{j}, j, k=1,2,3, \text { which are } \\
& \text { periodic in } E_{3}-\bigcup_{m}\{2 \pi m\}, \text { and in }
\end{aligned}
$$$$
L^{2}\left(T_{3}\right), L^{2}\left(T_{3}\right) \text { and } L^{1}\left(T_{3}\right) \text {, respectively. }
$$

$$
H^{\Upsilon}(0)=q_{j}^{\wedge}(0)=u_{j}^{k \wedge}(0)=0,
$$

and for $m \neq 0$,

$$
\begin{aligned}
& H^{\Upsilon}(m)=|m|^{-2}, \quad \hat{q_{j}}(m)=i m_{j}|m|^{-2}, \\
& v u_{j}^{k \wedge}(m)=\left[-\delta_{j}^{k}+m_{j} m_{k}|m|^{-2}\right]|m|^{-2},
\end{aligned}
$$

where $\delta_{j}^{k}$ is the Kronecker $\delta$.

To be specific, we define $H(x, t), q_{j}(x, t)$ and $u_{k}^{j}(x, t)$ for $t>0$ in a manner analogous to (2.43) using (2.47) and (2.48). Then in [5, p. 72], it is shown that the limits of $H(x, t)$ and $q_{j}(x, t)$ exist and are finite as $t \rightarrow 0$ for $x$ in $E_{3}-$ $\bigcup_{m}\{2 \pi m\}$. Defining $H(x)$ and $q_{j}(x)$, respectively, as these limits, it is shown 
furthermore in $[5$, p. 72$]$ that these functions have the properties enumerated in (2.46).

Setting $G(x)=-\Sigma_{m \neq 0} e^{i(m, x)}|m|^{-4}$ and observing that $\Delta G(x)=H(x)$ for $x$ in $E_{3}-\bigcup_{m}\{2 \pi m\}$, we see that $G$ is in $C^{\infty}\left(E_{3}-\bigcup_{m}\{2 \pi m\}\right)$. Defining $v u_{j}^{k}(x)=-\delta_{j}^{k} H(x)+\partial^{2} G(x) / \partial x_{j} \partial x_{k}$, we observe furthermore from the Riesz-Fischer theorem that $u_{j}^{k}$ does indeed have the properties enumerated in (2.46).

For $\mathbf{f}=\left(f_{1}, f_{2}, f_{3}\right)$ a continuous periodic vector in $E_{3}$ (i.e., $f_{j}$ is a continuous periodic function for $j=1,2,3)$, we shall set

$$
\omega\left(t, \mathbf{f}, T_{3}\right)=\sup _{|x-y| \leqslant t, x, y \text { in } E_{3}}|\mathbf{f}(x)-\mathbf{f}(y)|
$$

in a manner analogous to (2.13) for continuous periodic functions. In a similar manner, the definition that $f$ satisfies a Dini condition on $T_{3}$, as well as the notions of $\omega^{* *}(t, f, \gamma)$ and $C^{\omega^{* *}(t, f, \gamma)}\left(T_{3}\right)$ defined in (2.15) and (2.16), respectively, are carried over to vectors. Likewise, the notions of $C^{1+\omega^{* *}(t, f, \gamma)}\left(T_{3}\right)$ and $C^{2+\omega^{* *}}(t, \mathbf{f}, \gamma)$ are to be given the obvious interpretations.

The next lemma we establish is the following:

LEMMA 3. Let $\mathbf{f}=\left(f_{1}, f_{2}, f_{3}\right)$ be a continuous periodic vector defined in $E_{3}$ satisfying a Dini condition on $T_{3}$. Suppose also that $f_{k}^{\wedge}(0)=0, k=1,2,3$. For $j=1,2,3$, set

$$
v_{j}(x)=(2 \pi)^{-3} \sum_{k=1}^{3} \int_{T_{3}} u_{j}^{k}(y) f_{k}(x-y) d y
$$

and

$$
p(x)=(2 \pi)^{-3} \sum_{k=1}^{3} \int_{T_{3}} q_{k}(y) f_{k}(x-y) d y .
$$

Then $p$ and $v_{j}$ are continuous periodic functions in $E_{3}$ which are in $C^{1+\omega^{* *}(t, f, 1)}\left(T_{3}\right)$ and $C^{2+\omega^{* *}(t, f, 1)}\left(T_{3}\right)$, respectively. Furthermore,

$$
v \Delta v_{j}-\partial p / \partial x_{j}=f_{j}, \quad j=1,2,3
$$

$$
\sum_{j=1}^{3} \partial v_{j} / \partial x_{j}=0
$$

In order to establish the lemma, we set

$$
v_{j}^{k}(x)=(2 \pi)^{-3} \int_{T_{3}} u_{j}^{k}(y) f_{k}(x-y) d y
$$

and observe from the hypothesis of the lemma, (2.46), and (2.52) that

$$
v_{j}^{k} \text { is a continuous periodic function in } E_{3} \text {. }
$$

From (2.52), we next observe that for every lattice point $m$,

$$
v_{j}^{k \wedge}(m)=u_{j}^{k \wedge}(m) f_{k}^{\wedge}(m) .
$$


Now for $m \neq 0$, we have from (2.48) that

$$
v m_{\alpha} m_{\beta} u_{j}^{k \wedge}(m) f_{k}^{\wedge}(m)=-\delta_{j}^{k} m_{\alpha} m_{\beta}|m|^{-2} f_{k}^{\wedge}(m)+m_{\alpha} m_{\beta} m_{j} m_{k}|m|^{-4} f_{k}^{\wedge}(m) .
$$

Since by hypothesis, $f_{k}$ satisfies a Dini condition on $T_{3}$, we see from Lemma 2 that there is a function

$$
h_{\alpha \beta j k} \text { in } C^{\omega^{* *}\left(t, f_{k}, 1\right)}\left(T_{3}\right)
$$

such that

$$
m_{\alpha} m_{\beta} u_{j}^{k \wedge}(m) f_{k}^{\wedge}(m)=h_{\alpha \beta j k}^{\wedge}(m) \text { for every } m .
$$

We consequently conclude from Remark 2, (2.53), (2.54), (2.56), and (2.57) that

$$
v_{j}^{k} \text { is in } C^{2+\omega^{* *}\left(t, f_{k}, 1\right)}\left(T_{3}\right) \text {. }
$$

Since $\omega\left(t, f_{k}, T_{3}\right) \leqslant \omega\left(t, f, T_{3}\right)$, we conclude from (2.49), (2.52), and (2.58) that

$$
\begin{aligned}
& v_{j} \text { is a continuous periodic function in } \\
& C^{2+\omega^{* *}(t, f, 1)}\left(T_{3}\right) .
\end{aligned}
$$

A similar proof using $q_{\hat{k}}(m)$ and Remark 1 establishes that

$$
p \text { is a continuous periodic function in }
$$

$$
C^{1+\omega^{* *}(t, \mathrm{f}, 1)}\left(T_{3}\right) \text {. }
$$

Next, it follows from an easy computation, using (2.48), that

$$
\begin{aligned}
-\nu|m|^{2} \hat{v_{j}}(m)-i m_{j} \hat{p^{\wedge}(m)} & =f_{j}^{\hat{j}}(m), \quad j=1,2,3, \\
\sum_{j=1}^{3} i m_{j} v_{j}^{\hat{j}}(m) & =0
\end{aligned}
$$

for every lattice point $m$.

But then (2.51) follows immediately from (2.59), (2.60), (2.61), and the well-known uniqueness theorem for Fourier coefficients. The proof of Lemma 3 is therefore complete.

Next, we establish the following lemma which is essentially a corollary of Lemma 3.

LEMMA 4. Let $\mathbf{f}=\left(f_{1}, f_{2}, f_{3}\right)$ be a periodic vector in $C^{n}\left(E_{3}\right)$, $n$ a nonnegative integer. Suppose that for $k=1,2,3$, every partial derivative of order $n$ of $f_{k}$ satisfies a Dini condition on $T_{3}$, and suppose also that $f_{k}^{\wedge}(0)=0$. For $j=1$, 2,3 , let $v_{j}$ be defined by (2.49) and let $p$ be defined by (2.50). Thęn $p$ and $v_{j}$ are periodic functions in $C^{n+1}\left(E_{3}\right)$ and $C^{n+2}\left(E_{3}\right)$, respectively, and the pair $\mathrm{v}, p$ satisfies the system of equations in (2.51).

To prove Lemma 4, we proceed by induction. The case $n=0$ follows immediately from Lemma 3. Assume therefore that the lemma is true for $n \leqslant N$. To complete the proof of the lemma, it remains to show the following: 
If $f_{k}$ is in $C^{N+1}\left(E_{3}\right)$ for $k=1,2,3$, where $N$ is a nonnegative integer, and if every partial derivative of order $N+1$ of $f_{k}$ satisfies a Dini condition on $T_{3}$, then $p$ and $v_{j}$ are in $C^{N+2}\left(E_{3}\right)$ and $C^{N+3}\left(E_{3}\right)$, respectively.

Let $D$ represent a partial derivative of order $N+2$. Then the conclusion in (2.62) concerning $v_{j}$ will follow if we show

$$
D v_{j} \text { is in } C^{1}\left(E_{3}\right) \text {. }
$$

From (2.49), and the fact that $f_{k}$ is in at least $C^{1}\left(T_{3}\right)$, we see that for $\alpha=$ $1,2,3$,

$$
v_{j x_{\alpha}}=(2 \pi)^{-3} \sum_{k=1}^{3} \int_{T_{3}} u_{j}^{k}(y) f_{k x_{\alpha}}(x-y) d y .
$$

But now $f_{k x_{\alpha}}$ is in $C^{N}\left(E_{3}\right)$ and satisfies a Dini condition on $T_{3}$. Therefore, by the inductive assumption,

$$
v_{j x_{\alpha}} \text { is in } C^{N+2}\left(E_{3}\right) \text { for } \alpha, j=1,2,3 .
$$

But then it follows from (2.65) that $D v_{j x_{\alpha}}$ is in $C^{0}\left(E_{3}\right)$ for $\alpha, j=1,2,3$. This last fact implies that $D v_{j}$ is in $C^{1}\left(E_{3}\right)$, and (2.63) is established. Consequently, $v$ is in $C^{N+3}\left(E_{3}\right)$.

A similar proof shows that $p$ is in $C^{N+2}\left(E_{3}\right)$. Therefore (2.62) is established. Since the fact that the pair $\mathbf{v}, p$ satisfies the system in (2.51) follows immediately from Lemma 3 , we see that the proof of Lemma 4 is complete.

LEMMA 5. Let $\mathbf{f}=\left(f_{1}, f_{2}, f_{3}\right)$ be a periodic vector in $C^{n+\theta}\left(E_{3}\right), n$ a nonnegative integer and $0<\theta<1$. Suppose also that $\hat{f}_{k}^{\prime}(0)=0$. For $j=1,2,3$, let $v_{j}$ be defined by (2.50). Then $p$ and $v_{j}$ are periodic functions in $C^{n+1+\theta}\left(E_{3}\right)$ and $C^{n+2+\theta}\left(E_{3}\right)$, respectively, and the pair $\mathrm{v}, p$ satisfies the system of equations in (2.51).

To establish Lemma 5, we first of all deal with the case $n=0$. It follows from the hypothesis of the lemma that in this case there is a constant $A_{1}$ such that $\omega\left(t, \mathrm{f}, T_{3}\right) \leqslant A_{1} t^{\theta}$ for $0<t \leqslant 1$. Since $0<\theta<1$, we consequently obtain from (2.15) that there is a constant $A_{2}$ such that

$$
\omega^{* *}(t, \mathrm{f}, 1) \leqslant A_{2} t \text { for } 0<t \leqslant 1 \text {. }
$$

From the conclusion in Lemma 3, we have that $v_{j}$ is in $C^{2+\omega^{* *}}(t, f, 1)\left(T_{3}\right)$ for $j=1,2,3$. The fact that $v_{j}$ is also a continuous periodic function in conjunction with (2.66) shows that $v_{j}$ is in $C^{2+\theta}\left(E_{3}\right)$. In a similar manner, we have that $p$ is in $C^{1+\theta}\left(E_{3}\right)$. Therefore, the case $n=0$ of Lemma 5 is established.

We proceed by induction and assume Lemma 5 is true when $n \leqslant N, N$ a 
nonnegative integer. The proof of the lemma will be complete once we establish the following:

If $\mathbf{f}$ is in $C^{N+1+\theta}\left(E_{3}\right)$, then $p$ is in $C^{N+2+\theta}\left(E_{3}\right)$ and $v_{j}$ is in $C^{N+3+\theta}\left(E_{3}\right), j=1,2,3$.

From (2.67), we have that $\mathrm{f}$ is at least in $C^{\mathbf{1}}\left(E_{3}\right)$. Consequently, it follows from (2.49) that $v_{j x_{\alpha}}$ is given by (2.64) for $j, \alpha=1,2,3$. But $f_{k x_{\alpha}}$ is in $C^{N+\theta}\left(E_{3}\right)$. Consequently, by the inductive assumption, $v_{j x_{\alpha}}$ is in $C^{N+2+\theta}\left(E_{3}\right)$. This implies that $v_{j}$ is in $C^{N+3+\theta}\left(E_{3}\right)$, and (2.67) is established for $v_{j}, j=1,2$, 3. A similar approach shows that $p$ is in $C^{N+2+\theta}\left(E_{3}\right)$. (2.67) is therefore established, and the proof of Lemma 5 is complete.

Next, we state the following remark:

REMARK 3. Let $p$ and $v_{j}$ be, respectively, in $C^{0}\left[B\left(x^{0}, r_{0}\right)\right]$ and $C^{1}\left[B\left(x^{0}, r_{0}\right)\right]$, $j=1,2,3$. Suppose there are constants $A_{j}$ such that

$$
\int_{B\left(x^{0}, r_{0}\right)} \nu v_{j} \Delta \psi+p \frac{\partial \psi}{\partial x_{j}}=\int_{B\left(x^{0}, r_{0}\right)} A_{j} \psi d x
$$

$$
\text { for } j=1,2,3 \text { and } \psi \text { in } C_{0}^{\infty}\left[B\left(x^{0}, r_{0}\right)\right]
$$

Suppose, furthermore,

$$
\sum_{j=1}^{3} v_{j x_{j}}=0 \text { in } B\left(x^{0}, r_{0}\right) \text {. }
$$

Then $p$ and $v_{j}$ are in $C^{\infty}\left[B\left(x^{0}, r_{0}\right)\right], j=1,2,3$, and

$$
\nu \Delta v_{j}-\partial p / \partial x_{j}=A_{j}, \quad j=1,2,3 .
$$

Using the method of spherical means (or mollifiers) to further smooth $v_{j}$ and $p$, it can be easily shown from (2.68) and (2.69) that $p$ is harmonic in $B\left(x^{0}, r_{0}\right)$. Weyl's lemma, in conjunction with (2.68) then gives that $v_{j}$ is in $C^{\infty}\left[B\left(x^{0}, r_{0}\right)\right]$. (2.70) then follows from (2.68). We leave the filling in of these details to the reader and consider the proof of Remark 3 complete.

3. Proof of Theorem 1. We first of all observe that $f$ is bounded on every compact subset of $\Omega$. Consequently we infer from [2, pp. 79-81] that there is a pair $\mathrm{v}^{\prime}, p$, respectively, in $C^{1}(\Omega)$ and $C^{0}(\Omega)$ such that if $B$ is an open 3-ball with $\bar{B} \subset \Omega$, then

$$
\int_{B}\left[\nu v_{j}^{\prime} \Delta \psi+p \frac{\partial \psi}{\partial x_{j}}\right] d x=\int_{B} \psi\left(\sum_{k=1}^{3} v_{k}^{\prime} v_{j x_{k}}^{\prime}-f_{j}\right) d x
$$

for $j=1,2,3$ and all $\psi$ in $C_{0}^{\infty}(B)$;

$$
\sum_{k=1}^{3} v_{k x_{k}}^{\prime}=0 \text { in } B \text {. }
$$


Also,

$$
\mathbf{v}^{\prime}=\mathbf{v} \text { almost everywhere in } \Omega \text {. }
$$

Furthermore, if $\Omega_{1}$ is a subdomain of $\Omega$ such that $\bar{\Omega}_{1}$ is compact in $\Omega$,

$$
\mathbf{v}^{\prime} \text { is in } C^{1+\theta}\left(\Omega_{1}\right) \text {, and } p \text { is in } C^{\theta}\left(\Omega_{1}\right) \text { for } 0<\theta<1 \text {. }
$$

Let $\Omega_{1}$ be fixed and suppose $f$ is not identically constant in $\Omega_{1}$. Then setting $\omega^{*}\left(t, \mathrm{f}, \Omega_{1}\right)=\delta\left(\Omega_{1}\right)$ for $t \geqslant \delta\left(\Omega_{1}\right)$ and $\omega^{*}\left(0, \mathrm{f}, \Omega_{1}\right)=0$, we observe from (1.8) that $\omega^{*}\left(t, f, \Omega_{1}\right)$ is a continuous concave nondecreasing function on the interval $0 \leqslant t<\infty$. [In particular, $\omega^{*}\left(2 t, \mathrm{f}, \Omega_{1}\right) \leqslant 2 \omega^{*}\left(t, \mathrm{f}, \Omega_{1}\right)$.] As a consequence, it is not difficult to see that, in order to establish the theorem, it is sufficient to establish the following:

$$
\text { If } \bar{B}\left(x^{0}, r_{0}\right) \subset \Omega_{1} \text { with } 0<r_{0}<1 / 2 \text {, then }
$$

$$
\begin{aligned}
& \text { (a) } \mathrm{v}^{\prime} \text { is in } C^{2+\omega^{*}\left(t, \mathrm{f}, \Omega_{1}\right)}\left[B\left(x^{0}, r_{0}\right)\right] \text {, and } \\
& \text { (b) } p \text { is in } C^{1+\omega^{*}\left(t, f, \Omega_{1}\right)}\left[B\left(x^{0}, r_{0}\right)\right] \text {. }
\end{aligned}
$$

We now proceed to establish both parts of (3.4). With no loss in generality we can assume $x^{0}=0$. Also, since $\bar{B}\left(0, r_{0}\right) \subset \Omega_{1}$ with $0<r_{0}<1 / 2$, we can find $r_{1}, r_{2}$, and $r_{3}$ such that

$$
r_{0}<r_{1}<r_{2}<r_{3}<1 / 2 \text { and } \bar{B}\left(0, r_{3}\right) \subset \Omega_{1} \text {. }
$$

Next, we select a function $\lambda$ with the following properties:

$$
\lambda \text { is in } C_{0}^{\infty}\left[B\left(0, r_{3}\right)\right] \text {, }
$$

We then define for $j=1,2,3$,

$$
\begin{aligned}
g_{j} & =\lambda\left(\sum_{k=1}^{3} v_{k}^{\prime} v_{j x_{k}}^{\prime}-f_{j}\right) \quad \text { in } B\left(0, r_{3}\right), \\
& =0 \text { in } T_{3}-\bar{B}\left(0, r_{3}\right)
\end{aligned}
$$

and extend $g_{j}$ by periodicity to all of $E_{3}$;

From (3.3), (3.5), (3.8), and the hypothesis of the theorem, we see that

$$
\begin{aligned}
\mathrm{g}= & \left(g_{1}, g_{2}, g_{3}\right) \text { is a continuous periodic vector } \\
& \text { satisfying a Dini condition on } T_{3} .
\end{aligned}
$$

Next, for $j=1,2,3$, we set

$$
w_{j}=(2 \pi)^{-3} \sum_{k=1}^{3} \int_{T_{3}} u_{j}^{k}(y)\left[g_{k}(x-y)-g_{k}^{\hat{k}}(0)\right] d y
$$

and

$$
P(x)=(2 \pi)^{-3} \sum_{k=1}^{3} \int_{T_{3}} q_{k}(y)\left[g_{k}(x-y)-\hat{g_{k}}(0)\right] d y
$$


From (3.9) and Lemma 3, we infer immediately that

$P$ and $w_{j}$ are continuous periodic functions in $C^{1+\omega^{* *}(t, g, 1)}\left(T_{3}\right)$ and $C^{2+\omega^{* *}(t, \mathrm{~g}, 1)}\left(T_{3}\right)$, respectively.

Furthermore,

$$
\begin{aligned}
& \nu \Delta w_{j}-\partial P / \partial x_{j}=g_{j}-\hat{g_{j}}(0), \quad j=1,2,3, \\
& \sum_{k=1}^{3} w_{k x_{k}}=0 .
\end{aligned}
$$

But then from (3.1), (3.5), (3.7), (3.8), (3.12), and (3.13), we have

$$
\int_{B\left(0, r_{2}\right)}\left[\nu\left(v_{j}^{\prime}-w_{j}\right) \Delta \psi+(p-P) \frac{\partial \psi}{\partial x_{j}}\right] d x=\int_{B\left(0, r_{2}\right)} \psi \hat{g_{j}}(0) d x
$$

$$
\text { for } j=1,2,3 \text { and all } \psi \text { in } C_{0}^{\infty}\left[B\left(0, r_{2}\right)\right] \text {, }
$$

and

$$
\sum_{k=1}^{3}\left(v_{k}^{\prime}-w_{k}\right)_{x_{k}}=0 \text { in } B\left(0, r_{2}\right) \text {. }
$$

Since $v_{j}^{\prime}-w_{j}$ is in $C^{1}\left[B\left(0, r_{3}\right)\right], j=1,2,3$, and $p-P$ is $C^{0}\left[B\left(0, r_{3}\right)\right]$, we obtain immediately from (3.14) and Remark 3 that

$$
p-P \text { and } v_{j}^{\prime}-w_{j} \text { are in } C^{\infty}\left[B\left(0, r_{2}\right)\right] \text { for } j=1,2,3 .
$$

But then it follows, in particular, from (3.12) and (3.15) that $p$ is in $C^{1}\left[B\left(0, r_{1}\right)\right]$ and $v_{j}^{\prime}$ is in $C^{2}\left[B\left(0, r_{1}\right)\right]$ for $j=1,2,3$. Since $B\left(0, r_{1}\right)$ is the prototype of a ball whose closure is contained in $\Omega$, we conclude that

$$
p \text { is in } C^{1}(\Omega) \text {, and } v_{j}^{\prime} \text { is in } C^{2}(\Omega), \quad j=1,2,3 .
$$

From (3.1) and (3.16), we see that (i) and (ii) of Theorem 1 are established. To show part (iii) of Theorem 1 , namely (3.4) with $x^{0}=0$, we proceed as follows.

We first observe from (3.8) and the fact that $f_{j}$, $v_{j}^{\prime}$, and $v_{j x_{k}}^{\prime}$ are locally bounded in $\Omega$ that there is a constant $A_{1}$ such that

$$
\omega\left(t, g_{j}, T_{3}\right) \leqslant \omega\left[t, g_{j}, B\left(0, r_{3}\right)\right]+A_{1} \omega\left(t, \lambda, E_{3}\right) \text { for } 0<t<r_{3} \text {. }
$$

Next, from (3.7), (3.8), and (3.16), we see that there is a constant $A_{2}$ such that

$$
\begin{aligned}
\omega\left[t, g_{j}, B\left(0, r_{3}\right)\right] \leqslant & \omega\left[t, \mathrm{f}, B\left(0, r_{3}\right)\right]+A_{2} t \\
& \text { for } 0<t<r_{3} \text { and } j=1,2,3 .
\end{aligned}
$$

We consequently conclude from (1.8), (2.15), (3.17), (3.18), (3.6), and (3.5) that there is a constant $A_{3}$ such that 


$$
\begin{array}{r}
\omega^{* *}\left(t, g_{j}, 1\right) \leqslant \omega^{*}\left[t, \mathbf{f}, B\left(0, r_{3}\right)\right]+A_{3} t \log (1 / t) \\
\text { for } 0<t<r_{3} \text { and } j=1,2,3 .
\end{array}
$$

From (3.15) and (3.16) we see that there is a constant $A_{4}$ such that for $0<t<r_{1}$ and $\alpha, \beta, j=1,2,3$,

$$
\text { (a) } \omega\left[t, v_{j x_{\alpha} x_{\beta}}^{\prime}, B\left(0, r_{1}\right)\right] \leqslant \omega\left[t, w_{j x_{\alpha} x_{\beta}}, B\left(0, r_{1}\right)\right]+A_{4} t
$$

$$
\text { (b) } \omega\left[t, p_{x_{j}}, B\left(0, r_{1}\right)\right] \leqslant \omega\left[t, P_{x_{j}}, B\left(0, r_{1}\right)\right]+A_{4} t \text {. }
$$

We consequently conclude from (3.12), (3.19), (3.20), and the fact that $0<r_{1}<r_{3}<1 / 2$ that there is a constant $A_{5}$ such that

$$
\text { (a) } \omega\left[t, v_{j x_{\alpha} x_{\beta}}^{\prime}, B\left(0, r_{1}\right)\right] \leqslant A_{5}\left\{\omega^{*}\left[t, \mathbf{f}, B\left(0, r_{3}\right)\right]+t \log (1 / t)\right\} \text {, }
$$

$$
\text { (b) } \omega\left[t, p_{x_{j}}, B\left(0, r_{1}\right)\right] \leqslant A_{5}\left\{\omega^{*}\left[t, \mathrm{f}, B\left(0, r_{3}\right)\right]+t \log (1 / t)\right\}
$$

$$
\text { for } 0<t<r_{1} \text {. }
$$

Now by assumption $f$ is not identically constant in $\Omega_{1}$. Consequently, it is not difficult to see (e.g., see $[6$, p. $45,1.12]$ ) that there is a positive constant $A_{6}$ such that $\omega\left(t, \mathrm{f}, \Omega_{1}\right) \geqslant A_{6} t$ for $0<t<\delta\left(\Omega_{1}\right)$. But then it follows that there is another positive constant $A_{7}$ such that

$$
t \log (1 / t) \leqslant A_{7} \omega^{*}\left(t, \mathrm{f}, \Omega_{1}\right) \text { for } 0<t<r_{1} .
$$

From the fact that $0<r_{0}<r_{1}$ and that $\omega^{*}\left[t, \mathbf{f}, B\left(0, r_{3}\right)\right] \leqslant \omega^{*}\left(t, \mathbf{f}, \Omega_{1}\right)$ for $0<t<r_{0}$, we obtain as an immediate consequence of (3.21)(a) and (3.22) that $v_{j}^{\prime}$ is in $C^{2+\omega^{*}\left(t, \mathrm{f}, \Omega_{1}\right)}\left[B\left(0, r_{0}\right)\right]$. From (3.21)(b) and (3.22), we likewise obtain that $p$ is in $C^{1+\omega^{*}\left(t, f, \Omega_{1}\right)}\left[B\left(0, r_{0}\right)\right]$. We have therefore established (3.4), and the proof of Theorem 1 is complete.

4. Proof of Theorem 2. The proof of Theorem 2 for the case $n=0$ is almost identical with that given for parts (i) and (ii) of Theorem 1 . We therefore consider this case established and proceed by induction.

Thus, suppose Theorem 2 holds for the case $n \leqslant N, N$ a nonnegative integer, and assume that $\mathrm{f}$ is in $C^{N+1}\left(\Omega^{\prime}\right)$ and that the partial derivatives of order $N+1$ of $f_{k}$ satisfy a Dini condition locally in $\Omega^{\prime}$. It then follows from the inductive assumption that there is a pair $\mathbf{v}^{\prime}, p$ such that

$$
\mathbf{v}^{\prime} \text { is in } C^{N+2}\left(\Omega^{\prime}\right) \text {, and } p \text { is in } C^{N+1}\left(\Omega^{\prime}\right),
$$

and, furthermore, in $\Omega^{\prime}$, 


$$
\begin{aligned}
& \nu \Delta v_{j}^{\prime}-p_{x_{j}}=\sum_{k=1}^{3} v_{k}^{\prime} v_{j x_{k}}^{\prime}-f_{j}, \quad j=1,2,3, \\
& \sum_{k=1}^{3} v_{k x_{k}}^{\prime}=0 .
\end{aligned}
$$

Also,

$$
\mathbf{v}^{\prime}=\mathbf{v} \text { almost everywhere in } \Omega^{\prime} .
$$

To establish Theorem 2, it is sufficient, therefore, to show the following:

$$
\text { If } \bar{B}\left(x^{0}, r_{0}\right) \subset \Omega^{\prime} \text { with } 0<r_{0}<1 / 2 \text {, then }
$$
(a) $\mathrm{v}^{\prime}$ is in $C^{N+3}\left[B\left(x^{0}, r_{0}\right)\right]$, and
(b) $p$ is in $C^{N+2}\left[B\left(x^{0}, r_{0}\right)\right]$.

We now proceed to establish both parts of (4.4). With no loss in generality, we can assume $x^{0}=0$. Also, since $\bar{B}\left(0, r_{0}\right) \subset \Omega^{\prime}$ with $0<r_{0}<1 / 2$, we can find $r_{1}, r_{2}$, and $r_{3}$ such that

$$
r_{0}<r_{1}<r_{2}<r_{3}<1 / 2 \text { and } \bar{B}\left(0, r_{3}\right) \subset \Omega^{\prime} .
$$

Next, we select a function $\lambda$ with the properties enumerated in (3.6) and (3.7) and define the function $g_{j}$ in $T_{3}$ for $j=1,2,3$ by (3.8). We then extend the function $g_{j}$ by periodicity to all of $E_{3}$.

From the fact that $\mathrm{f}$ is in $C^{N+1}\left(\Omega^{\prime}\right)$ and from (4.1), (4.5), and (3.8), we observe that

$$
g_{j} \text { is in } C^{N+\theta}\left(E_{3}\right) \text { for } 0<\theta<1 .
$$

Next, for $j=1,2,3$, and $x$ in $E_{3}$ we define $w_{j}(x)$ by (3.10) and $P(x)$ by (3.11). But then it follows from Lemma 5 and (4.6) that

$$
w_{j} \text { is in } C^{N+2+\theta}\left(E_{3}\right) \text {, and } P \text { is in } C^{N+1+\theta}\left(E_{3}\right)
$$

and, furthermore, in $E_{3}$,

$$
\begin{gathered}
\nu \Delta w_{j}-P_{x_{j}}=g_{j}-\hat{g_{j}}(0), \\
\sum_{k=1}^{3} w_{k x_{k}}=0 .
\end{gathered}
$$

Since $\lambda=1$ in $B\left(0, r_{2}\right)$, we see from (3.8) that $g_{j}=\Sigma_{k=1}^{3} v_{k}^{\prime} v_{k x_{j}}^{\prime}-f_{j}$ in $B\left(0, r_{2}\right)$. Consequently, we obtain from (4.2) and (4.8) that, in $B\left(0, r_{2}\right)$,

$$
\begin{gathered}
\nu \Delta\left(v_{j}^{\prime}-w_{j}\right)-(p-P)_{x_{j}}=\hat{g_{j}}(0), \\
\sum_{k=1}^{3}\left(v_{j}^{\prime}-w_{j}\right)_{x_{k}}=0 .
\end{gathered}
$$

It follows, therefore, from Remark 3 and (4.9) that $v_{j}-w_{j}$ is in $C^{\infty}\left[B\left(0, r_{2}\right)\right]$. But then from (4.7) and the fact that $r_{1}<r_{2}$, we obtain 


$$
v_{j} \text { is in } C^{N+2+\theta}\left[B\left(0, r_{1}\right)\right]
$$

Next, we choose $\eta$ such that

$$
\eta \text { is in } C_{0}^{\infty}\left[B\left(0, r_{1}\right)\right]
$$

and

$$
\eta=1 \text { in } B\left(0, r_{0}\right) \text {. }
$$

We define for $j=1,2,3$,

$$
\begin{aligned}
g_{j}^{\prime} & =\eta\left(\sum_{k=1}^{3} v_{k}^{\prime} v_{j x_{k}}^{\prime}-f_{j}\right) \quad \text { in } B\left(0, r_{1}\right) \\
& =0 \quad \text { in } T_{3}-B\left(0, r_{1}\right)
\end{aligned}
$$

and extend $g_{j}^{\prime}$ by periodicity to all of $E_{3}$.

Now, by assumption, $f_{k}$ is in $C^{N+1}\left(\Omega^{\prime}\right)$ and its partial derivatives of order $N+1$ satisfy a Dini condition locally in $\Omega^{\prime}$. Consequently, it follows from (4.10) through (4.13) that

$$
\begin{aligned}
& g_{j}^{\prime} \text { is a periodic function } C^{N+1}\left(E_{3}\right) \text { and every partial derivative } \\
& \text { of order } N+1 \text { of } g_{j}^{\prime} \text { satisfies a Dini condition on } T_{3} .
\end{aligned}
$$

Next, with $g_{j}^{\prime}$ replacing $g_{j}$, we define $w_{j}^{\prime}(x)$ by $(3.10)$ and $P^{\prime}(x)$ by (3.11). It follows immediately, therefore, from (4.14) and Lemma 4 that

$$
w_{j}^{\prime} \text { is in } C^{N+3}\left(E_{3}\right) \text {, and } P^{\prime} \text { is in } C^{N+2}\left(E_{3}\right)
$$

and, furthermore, that, in $E_{3}$,

$$
\begin{aligned}
& \Delta w_{j}^{\prime}-P_{x_{j}}^{\prime}=g_{j}^{\prime}-g_{j}^{i \wedge}(0), \\
& \sum_{k=1}^{3} w_{k x_{k}}^{\prime}=0 .
\end{aligned}
$$

But from (4.11) through (4.13), $g_{j}^{\prime}=\Sigma_{k=1}^{3} v_{k}^{\prime} v_{j x_{k}}^{\prime}-f_{j}$ in $B\left(0, r_{0}\right)$. Therefore, we conclude once again from (4.2), Remark 3 , and this time (4.16) that

$$
\begin{aligned}
& v_{j}^{\prime}-w_{j}^{\prime} \text { is in } C^{\infty}\left[B\left(0, r_{0}\right)\right], \text { and } \\
& p-p^{\prime} \text { is in } C^{\infty}\left[B\left(0, r_{0}\right)\right] .
\end{aligned}
$$

(4.17) in conjunction with (4.15) gives both parts of (4.4), and the proof of Theorem 2 is complete.

In closing, we would like to point out that using a fixed function $h$ satisfying a Dini condition locally in $\Omega^{\prime}$ and assuming that $f$ is in $C^{n+\omega\left(t, h, \Omega^{\prime}\right)}\left[\Omega^{\prime \prime}\right]$ where $\Omega^{\prime \prime}$ is a subdomain with compact closure in $\Omega^{\prime}$, it is not difficult to obtain for Theorem 2 an analogue of part (iii) of Theorem 1. We leave this for the interested reader. 


\section{REFERENCES}

1. A. P. Calderón and A. Zygmund, Singular integrals and periodic functions, Studia Math. 14 (1954), 249-271 (1955). MR 16, 1017.

2. H. Fujita, On the existence and regularity of the steady-state solutions of the NavierStokes equations, J. Fac. Sci. Univ. Tokyo Sect. 19 (1961), 59-102. MR 24 \#A2152.

3. E. W. Hobson, The theory of spherical harmonics, Cambridge Univ. Press, Cambridge, 1931.

4. O. A. Ladyženskaja, Mathematical problems in the dynamics of a viscous incompressible fluid, Fizmatgiz, Moscow, 1961; English transl., The mathematical theory of viscous imcompressible flow, 2nd rev. ed., Mathematics and its Applications, vol. 2, Gordon and Breach, New York, 1969. MR 27 \#5034a, b; 40 \#7610.

5. V. L. Shapiro, Fourier series in several variables, Bull. Amer. Math. Soc. 70 (1964), 48-93. MR 28 \#1448.

6. A. Zygmund, Trigonometric series. Vol. I, 2nd ed., Cambridge Univ. Press, New York, 1959. MR 21 \#6498.

DEPARTMENT OF MATHEMATICS, UNIVERSITY OF CALIFORNIA, RIVERSIDE, CALIFORNIA 92502 